\title{
La nueva normalidad de la realidad educativa en el contexto ecuatoriano
}

Fecha de recepción: 2021-08-15 • Fecha de aceptación: 2021-10-22 • Fecha de publicación: 2021-12-01

Franklin Gregorio Macias Arroyo ${ }^{1}$ Universidad Estatal de Milagro, Ecuador gmaciasa@unemi.edu.ec

https://orcid.org/0000-0002-0794-7542

Lorgia Patricia Astudillo Erazo ${ }^{2}$ Escuela de Educación Básica "Dr. Oswaldo Hurtado Larrea", Ecuador patricia230882@hotmail.com https://orcid.org/0000-0002-9732-2289

Silvino Alejandro Macías Arroyo ${ }^{3}$ Escuela de Educación Básica "Alfredo Pérez Guerrero", Ecuador samacias28@gmail.com https://orcid.org/0000-0001-9245-4808

\section{Resumen}

El sistema educativo diseñado para hacer frente a la era del COVID-19 está marcado por una nueva normalidad, en la que la digitalización impone formas de trabajar y aprender. Impulsa la educación hacia la tecnificación, un desarrollo que ya está en marcha, impulsado por el comercialismo y la ideología de mercado reinante. Por lo tanto, este artículo tiene como objetivo conocer la relación entre la nueva normalidad en la realidad educativa en el Ecuador, desde la perspectiva de los hogares ecuatorianos. En este estudio se utilizó el enfoque cuantitativo descriptivo, la técnica utilizada fue un muestreo aleatorio, se aplicó un cuestionario para identificar la nueva normalidad del hecho educativo en el Ecuador, mediante cuatro indicadores: (1) forma de impartir clases por 
parte de los docentes, (2) forma de evaluar clases por parte de los docentes, (3) plataformas para transmitir clases y (4) tecnologías para desarrollar clases. Se concluye que, si bien se han realizado varios estudios, la educación necesita ser explorada más a fondo. El cambio notorio en la forma de impartir y evaluar las clases, requiere una intervención a nivel de políticas para mejorar esta situación, además de exploraciones e investigaciones adicionales sobre la pedagogía eficaz para la enseñanza y el aprendizaje.

Palabras clave: nueva normalidad, realidad educativa, forma de evaluar, forma de impartir clases, plataformas digitales, tecnologías digitales

\begin{abstract}
The education system designed to cope with the COVID-19 era is marked by a new normality, in which digitalization imposes ways of working and learning. It pushes education towards technification, a development that is already underway, driven by commercialism and the prevailing market ideology. Therefore, this article aims to know the relationship between the new normality in the educational reality in Ecuador, from the perspective of Ecuadorian households. In this study we used the descriptive quantitative approach, the technique used was random sampling, a questionnaire was applied to identify the new normality of the educational fact in Ecuador, through four indicators: (1) way of teaching classes by teachers, (2) way of evaluating classes by teachers, (3) platforms to transmit classes and (4) technologies to develop classes. It is concluded that, although several studies have been conducted, education needs to be further explored. The noticeable change in the way classes are taught and evaluated requires policy-level intervention to improve this situation, as well as further exploration and research on effective pedagogy for teaching and learning.
\end{abstract}

Keywords: new normal, educational reality, way of evaluating, way of teaching, digital platforms, digital technologies 


\section{Introducción}

Durante la pandemia, la mayoría de los países del mundo han cerrado temporalmente las instituciones educativas para contener la propagación de la pandemia COVID-19 y reducir las infecciones, este cierre ha afectado a más de 1200 millones de estudiantes en todo el mundo, debido al cierre de la comunidad y la cuarentena de varios países, lo que ha llevado a estudiantes y docentes a estudiar y trabajar desde casa, y por ende, el uso de plataformas de aprendizaje en línea (Tria, 2020). Sin embargo, la implementación del aprendizaje en línea planteó diferentes riesgos, problemas y desafíos, tanto para los docentes, como para los estudiantes (Plá et al., 2020).

La pandemia de COVID-19 ha cambiado la vida cotidiana, ha dejado como resultado enfermedades y muertes a gran escala, y ha provocado medidas preventivas como el distanciamiento social, el confinamiento y el cierre de escuelas. Ha golpeado de manera desproporcionada a quienes brindan servicios esenciales y a quienes no pueden trabajar a distancia; en un mercado ya precario, el desempleo está teniendo terribles consecuencias (Pacheco, 2020)social, and economic spheres. Are there continuities as well? Based on a literature review (primarily of UNESCO and OECD publications and their critics. La pandemia es ahora el principal signo de globalización y desglobalización, ha interrumpido el paso del tiempo y ha deshecho lo que era normal. Es el surgimiento de un evento que reinicia el tiempo, crea rupturas y desequilibrios radicales, y provoca una contingencia que se convierte en una nueva necesidad.

El sistema educativo, diseñado para hacer frente a la era de COVID-19, está marcado por una nueva normalidad. Esta expresión apareció inicialmente en el área empresarial, la frase se utiliza para recordar a los economistas que deben aumentar su confianza en que la economía industrial volverá a la normalidad, después de una recesión (Acuña, 2021). Este término se usa en diferentes contextos, para indicar el cambio de algo no típico en típico. La nueva normalidad, como situación después de que ocurran algunos cambios intensos, reemplaza el estado habitual aceptado después de que han ocurrido ciertos eventos (Andarwulan et al., 2021)mainly for elementary school teachers. This study aims to investigate the readiness of elementary school teachers towards the adoption of online-based learning policies during Covid-19. There are six indicators to measure teacher readiness, they are (1.

La pandemia marca el comienzo de una nueva normalidad, en la que la digitalización impone formas de trabajar y aprender. Impulsa la educación hacia la tecnificación, un desarrollo que ya está en marcha, impulsado por el comercialismo y la ideología de mercado reinante. Pacheco (2020)social, and economic spheres. Are there continuities as well? Based on a literature review (primarily of UNESCO and OECD publications and their critics señala que muchas instituciones tenían planes para hacer un mayor uso de la tecnología en la enseñanza, pero el brote de COVID-19 ha significado que los cambios que se pretendían producir durante meses o años, debían implementarse en unos pocos días.

Dentro de la nueva normalidad, la situación presenta un desafío único al proceso de toma de decisiones de cada líder educativo. Por lo tanto, para mantener la calidad de la enseñanza en 
todas las escuelas, este artículo tiene como objetivo conocer la relación entre la nueva normalidad en la realidad educativa en el Ecuador, desde la perspectiva de los hogares ecuatorianos, en tal sentido, el estudio tiene un gran interés en las tendencias actuales y futuras de la nueva educación normal.

El aprendizaje basado en tecnología o en línea surge como la opción para implementar el proceso de enseñanza y aprendizaje en la nueva era normal, la implementación de este aprendizaje en realidad no es algo nuevo. Antes de la era del distanciamiento social, muchas instituciones educativas implementaron el aprendizaje en línea o el aprendizaje mixto (Andarwulan et al., 2021) mainly for elementary school teachers. This study aims to investigate the readiness of elementary school teachers towards the adoption of online-based learning policies during Covid-19. There are six indicators to measure teacher readiness, they are (1. Pese a esto, dado que las escuelas han estado cerradas debido a la cuarentena, el proceso de aprendizaje se realiza en su mayor parte, o en su totalidad, utilizando el sistema en línea. Este aprendizaje puede aumentar la creatividad y la independencia de los estudiantes, los docentes pueden innovar en el uso de las redes sociales y aplicaciones de aprendizaje para acercarse a los estudiantes, lo cual también se puede implementar como un medio alternativo en el proceso de aprendizaje, cuando las actividades de aprendizaje no se pueden realizar a través de interacciones cara a cara, además, los estudiantes tienen más posibilidades de acceso a muchas fuentes de información en sitios web de aprendizaje (Tejedor et al., 2020).

En Ecuador, la política de aprendizaje en línea en la nueva era normal se está convirtiendo en un ideal que es difícil de hacer. En situaciones normales, ocurren muchos obstáculos al implementar el aprendizaje en línea, por lo que es necesario preparar factores para respaldar este logro, tanto del docente, como de los estudiantes, como la suficiencia económica, la disponibilidad de instalaciones e infraestructura adecuadas, redes de Internet y habilidades para implementar la tecnología (Tejedor et al., 2020). Los puntos importantes que determinan el logro del aprendizaje en línea serían los recursos educativos en el hogar, la disponibilidad de espacio, la educación a nivel de los padres, la fluidez de los padres en el idioma de la enseñanza escolar, la competencia digital de los padres y la participación de los padres con la institución (Vargas Jiménez, 2020).

\subsection{Fortalecimiento de las plataformas de aprendizaje en línea}

Diferentes países de todo el mundo han introducido varias respuestas durante la pandemia para continuar el proceso educativo como la introducción del aprendizaje a distancia. Se trata de plataformas de aprendizaje en línea, se implantaron transmisiones de TV, pautas, recursos, videoconferencias y canales en línea (Prince, 2021). Para las instituciones que reabrirán las puertas, es vital planificar cómo podrán seguir su misión de implementar una educación de calidad para todos los estudiantes. Dado que es un lugar público donde la multitud es inevitable, y los estudiantes son vulnerables, es necesario fortalecer las políticas en términos de impartición de educación, para brindar oportunidades para plataformas de aprendizaje en línea.

Los diferentes sectores de aprendizaje han propuesto numerosos programas innovadores, del mismo modo, la nueva normalidad serían las aulas virtuales, fortaleciendo las plataformas en línea y el aprendizaje combinado. Además, las instituciones educativas adoptarán numerosas opciones 
para la enseñanza, el aprendizaje presencial, combinado, el aprendizaje a distancia y la educación en el hogar y otros modos de enseñanza (Acosta, 2020). Sin embargo, la implementación proyectaría tales problemas a los estudiantes que tienen acceso limitado a Internet, no tienen dispositivos y no cuentan con los recursos económicos necesarios. A pesar de lo mencionado, existen desafíos que serían brechas de equidad, la seguridad de los estudiantes, la calidad del aprendizaje comprometida y los malos resultados de las evaluaciones (Plá et al., 2020). Además, las actividades de laboratorio en ciencias y otras materias que requieren desempeño, como educación física, cultura y artes, se limitarían a pruebas de papel y lápiz, a menos que las estudiantes requieran que los estudiantes estén físicamente presentes para ser evaluados mediante pruebas de desempeño.

Conjuntamente, las actividades extracurriculares como exploración, graduación, deportes, concursos, entre otros., se reducirán o suspenderán. En cuanto a la docencia, también se recomienda la formación del profesorado a la instrucción en línea, el aprendizaje mixto y el aprendizaje a distancia para adecuarse al nuevo formato instruccional (Tria, 2020). En este sentido, deben reforzarse las competencias de los docentes, tanto en pedagogía, como en tecnología, esta transición a la nueva normalidad, desde las cuatro esquinas del aula, hasta las fronteras de la realidad virtual, cada institución de aprendizaje debe estudiar qué tan exitoso es el aprendizaje en línea al brindar educación de calidad y educación basada en resultados a los estudiantes (Acuña, 2021).

\section{Metodología}

En este estudio se utilizó el método cuantitativo/descriptivo. Este se eligió para identificar la nueva normalidad del hecho educativo en el Ecuador, desde la perspectiva de los hogares ecuatorianos. El propósito del diseño de la investigación fue describir e interpretar el estado actual de un individuo, entorno, condición o evento (Hernández-Sampieri \& Mendoza, 2018). Con métodos de investigación descriptiva/cuantitativa, este estudio tiene como objetivo examinar y definir la situación existente (Hernández Sampieri et al., 2014), al establecer este método se analizó el puntaje de cada variable.

La población es un área generadora que está formada por objetos que tienen ciertas cualidades y características determinadas por los investigadores a estudiar y las conclusiones a extraer (Guillermina, 2014). La muestra de esta investigación son los representantes de los hogares ecuatorianos que se extendieron en 22 ciudades del Ecuador. Este grupo se selecciona porque es el mayor representante de la educación en casa, bajo la nueva normalidad. La técnica utilizada en este estudio es un muestreo aleatorio de 4589 representantes, que fueron seleccionados del nivel de educación básica como bachillerato.

La técnica de recopilación de datos de investigación se realizó mediante un cuestionario para identificar la nueva normalidad del hecho educativo en el Ecuador, lo cual se midió mediante cuatro indicadores, tales como (1) forma de impartir clases por parte de los docentes, (2) forma de evaluar clases por parte de los docentes, (3) plataformas para transmitir clases y (4) tecnologías para desarrollar clases. 
Los datos se recopilaron con un cuestionario en línea debido a la facilidad en el contexto actual, para poder responder. Además, también fue fácil de administrar y acceder a través de varios dispositivos. La mayoría de los participantes fueron contactados a través de grupos de WhatsApp y correo electrónico, mientras que otros fueron acercados a través de una carta directa hacia las instituciones educativas y correo electrónico personal. El enlace de los cuestionarios está alojado en la plataforma Google Forms, el mismo que estuvo abierto durante 3 semanas.

Las preguntas de investigación se examinaron con un análisis estadístico descriptivo y correlacional. En cuanto al descriptivo, se calcularon las desviaciones medias y estándar de las respuestas para todos los ítems de preparación y se presentaron gráficos. Para el segundo se empleó el coeficiente de correlación Tau - B de Kendall, ya que se utilizó para comprender la fuerza de la relación entre dos variables.

\section{Resultados}

La pandemia por COVID-19 ha causado importantes interrupciones en las actividades académicas. Esta investigación exploró la nueva normalidad en la experiencia de aprendizaje en los hogares ecuatorianos.

Los resultados han demostrado que la frecuencia del cambio de la forma de impartir clases por parte de docentes fue considerable, casi la mitad de los encuestados $(40 \%)$ consideraron que el cambio fue entre un 90 a $100 \%$ y $39 \%$ describió que el cambio estimado de dar clases fue entre un 60 a $89 \%$ (ver Figura 1).

\section{Figura 1.}

Forma de impartir clases por parte de docentes

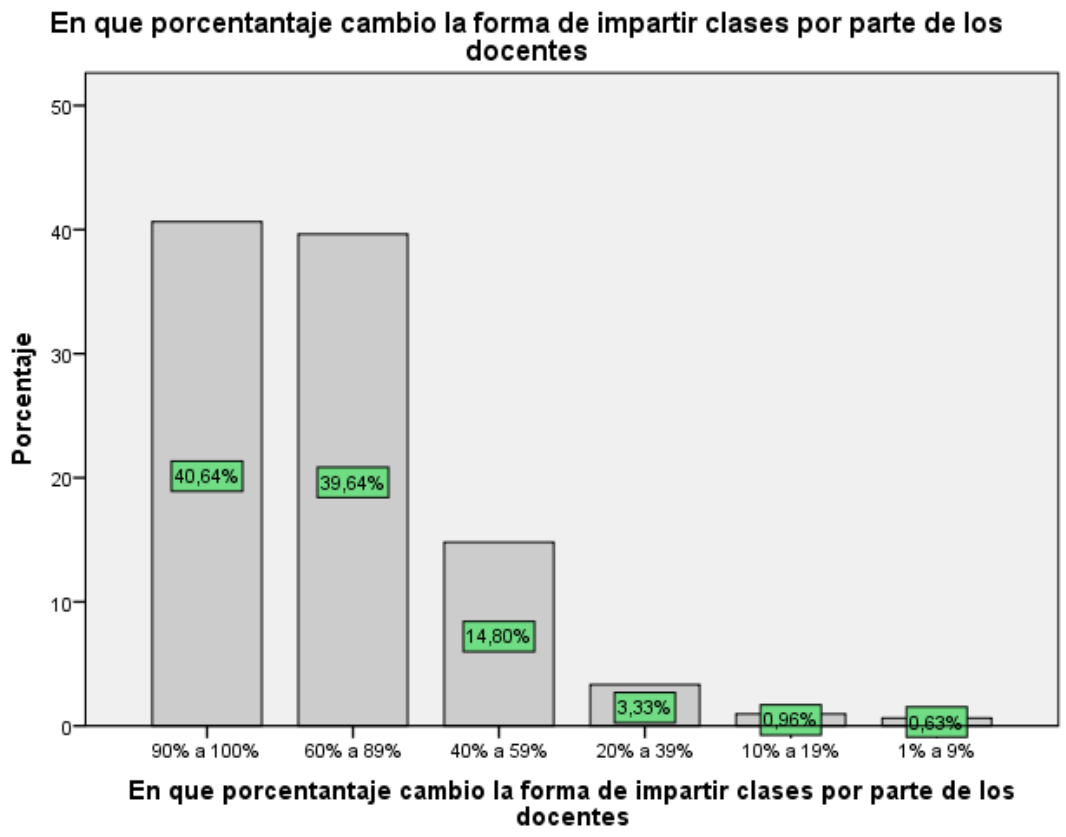


Al estudiar en qué porcentaje cambio la forma de evaluar las clases por parte de los docentes, en la Figura 2 se muestran estos resultados. En el gráfico se muestra que el $41,64 \%$ consideraron un cambio del 90 al $100 \%$, seguidos del $37 \%$, con una percepción de cambio del 60 al $89 \%$ y el $15 \%$ con un cambio del 40 al $59 \%$.

Figura 2.

Forma de evaluar clases por parte de docentes

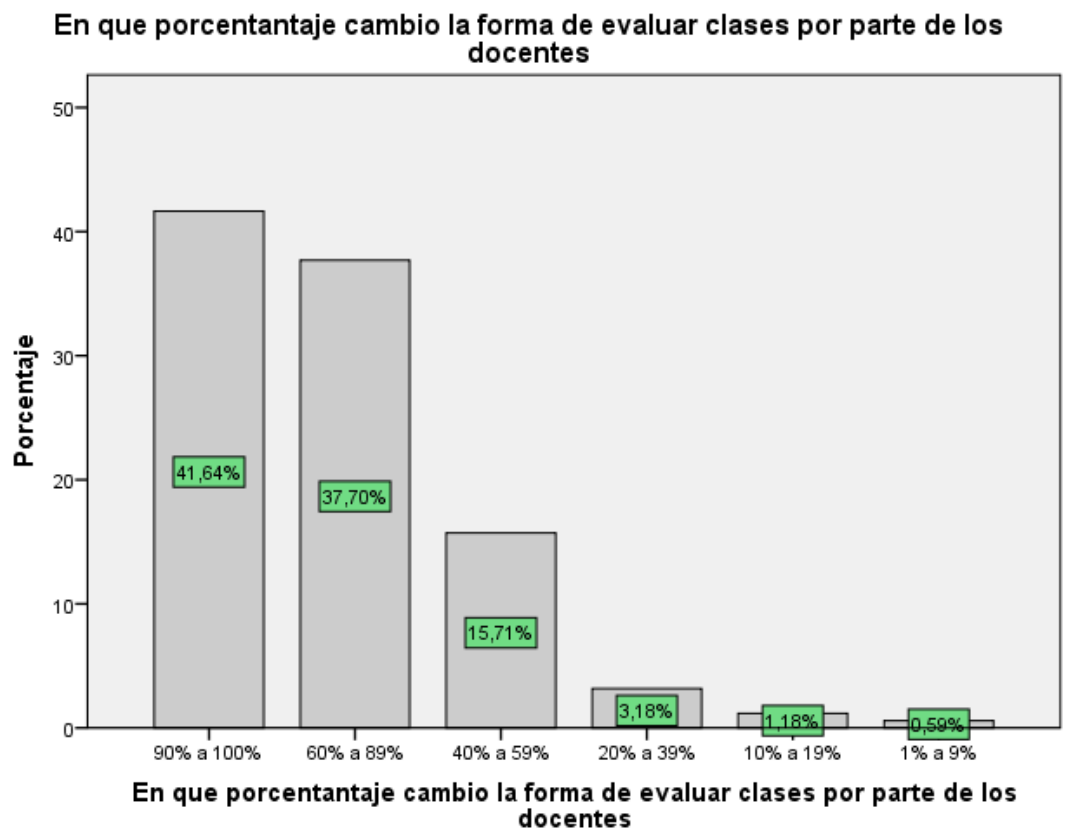

El estudio tenía una pregunta sobre plataformas para transmitir clases como parte de la nueva normalidad (Figura 3). Los resultados mostraron que las más utilizadas fueron Zoom $(40 \%)$ y Whatssap (38\%), seguidas de otras (17\%), Suite Google $(1.1 \%)$ y Youtube $(0.9 \%)$ plataformas que tuvieron significancias notorias. 
Figura 3.

Plataformas para transmitir clases

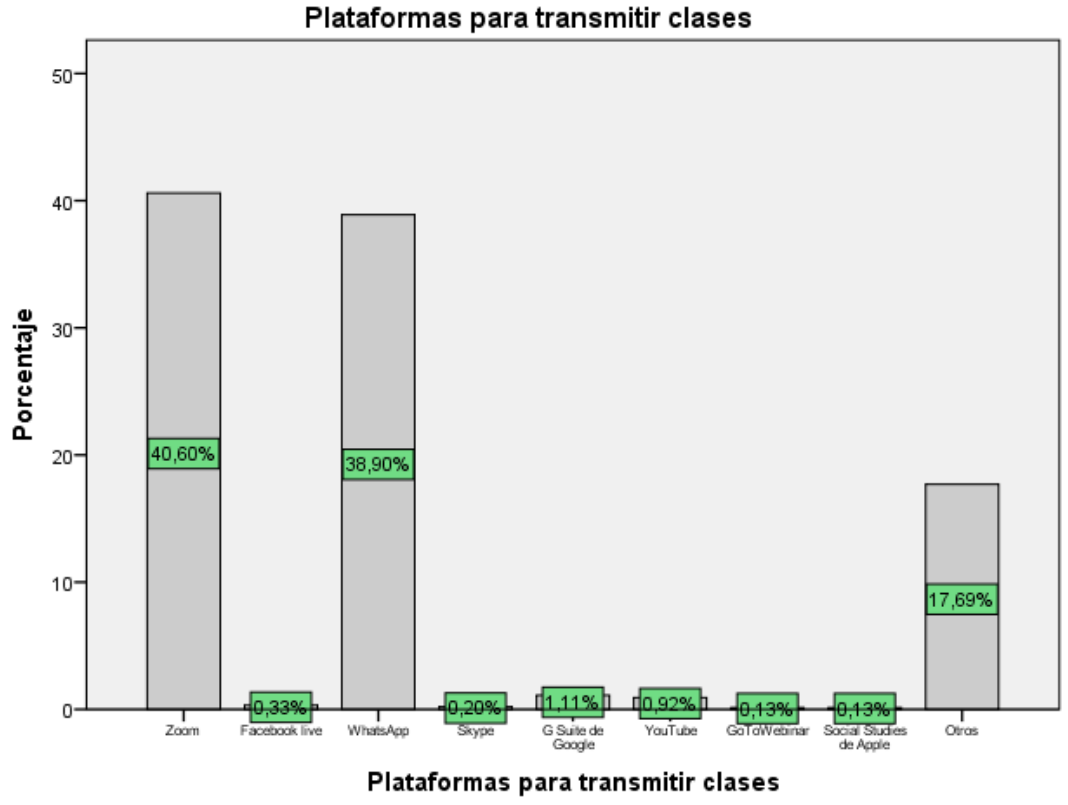

Figura 4.

Tecnologías para desarrollar clases

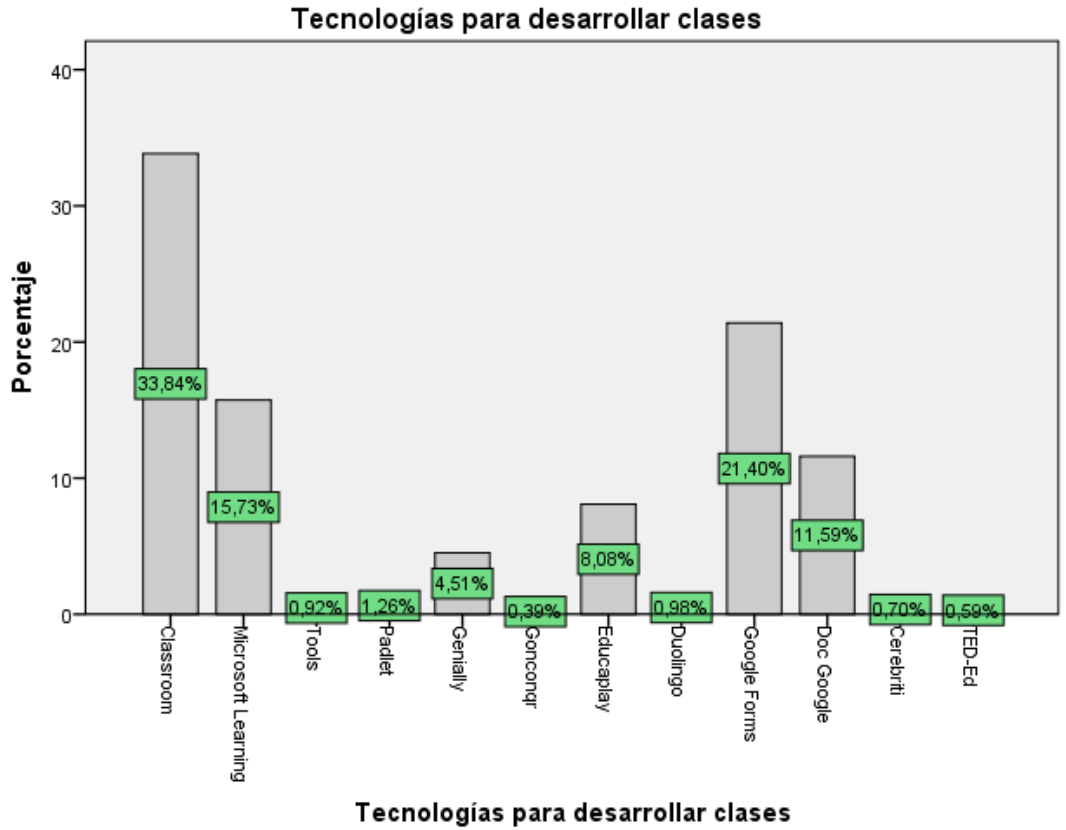


En cuanto a las tecnologías para el desarrollo de las clases, casi el 35\% ha utilizado classroom en las clases, seguido del $21 \%$ que ha utilizado Google Forms y el $15 \%$ ha utilizado Microsoft Learnings, y solo el $0,5 \%$ ha usado TED-Ed, tal como se observa en la Figura 4.

Para conocer la relación entre la nueva normalidad en la realidad educativa en el Ecuador, desde la perspectiva de los hogares ecuatorianos, se aplicó el método estadístico Tau B de Kendall, ya que es una medida no paramétrica de relaciones entre columnas de datos clasificados, el coeficiente de correlación de Tau devuelve un valor de 0 a 1, donde 0 no es una relación y es 1 es una relación perfecta.

En tal sentido, se obtuvo que únicamente existe relación entre el porcentaje cambio la forma de impartir clases por parte de los docentes y el porcentaje cambio la forma de clases por parte de los docentes, con un coeficiente de 0.64 (Tabla 1), dicho valor determina que existe una relación considerable entre la forma de evaluar las clases y el cambio de la modalidad de impartir clases.

Tabla 1.

La nueva normalidad en la realidad educativa

\begin{tabular}{|c|c|c|c|c|c|c|}
\hline \multicolumn{7}{|c|}{ Correlaciones } \\
\hline & & & $\begin{array}{l}\text { En qué } \\
\text { porcentaje } \\
\text { cambio la } \\
\text { forma de } \\
\text { impartir clases } \\
\text { por parte de } \\
\text { los docentes }\end{array}$ & $\begin{array}{l}\text { En qué } \\
\text { porcentaje } \\
\text { cambio la } \\
\text { forma de } \\
\text { evaluar clases } \\
\text { por parte de } \\
\text { los docentes }\end{array}$ & $\begin{array}{l}\text { Plataformas } \\
\text { para transmitir } \\
\text { clases }\end{array}$ & $\begin{array}{l}\text { Tecnologías } \\
\text { para } \\
\text { desarrollar } \\
\text { clases }\end{array}$ \\
\hline \multirow[t]{12}{*}{$\begin{array}{l}\text { Tau_b de } \\
\text { Kendall }\end{array}$} & \multirow{3}{*}{$\begin{array}{l}\text { En qué porcentaje } \\
\text { cambio la forma } \\
\text { de impartir clases } \\
\text { por parte de los } \\
\text { docentes }\end{array}$} & $\begin{array}{l}\text { Coeficiente } \\
\text { de correlación }\end{array}$ & 1,000 &, $647^{\star *}$ &,$- 049^{\star \star}$ &,- 006 \\
\hline & & Sig. (bilateral) & . & ,000 & ,000 & ,605 \\
\hline & & $\mathrm{N}$ & 4589 & 4589 & 4589 & 4589 \\
\hline & \multirow{3}{*}{$\begin{array}{l}\text { En qué porcentaje } \\
\text { cambio la forma } \\
\text { de evaluar clases } \\
\text { por parte de los } \\
\text { docentes }\end{array}$} & $\begin{array}{l}\text { Coeficiente } \\
\text { de correlación }\end{array}$ & $647^{* *}$ & 1,000 &,- 020 & 001 \\
\hline & & Sig. (bilateral) & ,000 & . & , 130 & ,904 \\
\hline & & $\mathrm{N}$ & 4589 & 4589 & 4589 & 4589 \\
\hline & \multirow[t]{3}{*}{$\begin{array}{l}\text { Plataformas para } \\
\text { transmitir clases }\end{array}$} & $\begin{array}{l}\text { Coeficiente } \\
\text { de correlación }\end{array}$ &,$- 049^{\star *}$ &,- 020 & 1,000 &, $086^{* *}$ \\
\hline & & Sig. (bilateral) & ,000 & , 130 & . &, 000 \\
\hline & & $\mathrm{N}$ & 4589 & 4589 & 4589 & 4589 \\
\hline & \multirow[t]{3}{*}{$\begin{array}{l}\text { Tecnologías para } \\
\text { desarrollar clases }\end{array}$} & $\begin{array}{l}\text { Coeficiente } \\
\text { de correlación }\end{array}$ &,- 006 & ,001 &, $086^{* *}$ & 1,000 \\
\hline & & Sig. (bilateral) & ,605 & ,904 & ,000 & \\
\hline & & $\mathrm{N}$ & 4589 & 4589 & 4589 & 4589 \\
\hline
\end{tabular}




\section{Conclusiones}

El estudio sobre la nueva realidad en la educación generada por la pandemia COVID-19, concluye que, si bien se han realizado varios estudios, la educación necesita ser explorada más a fondo. El cambio notorio en la forma de impartir y evaluar las clases requiere una intervención a nivel de políticas para mejorar esta situación, además de exploración e investigación adicionales sobre la pedagogía eficaz para la enseñanza y el aprendizaje.

Además, se encuentra la necesidad del uso de plataformas y herramientas digitales para evaluaciones auténticas y retroalimentación oportuna en otra área de estudio. La asequibilidad y accesibilidad para todos los estudiantes de diversos antecedentes económicos se infiere que es un desafío, para el cual el desarrollador de herramientas educativas podría enfocarse en la personalización. Como punto final, en la relación entre la nueva normalidad en la realidad educativa en el Ecuador, únicamente se evidenció entre el porcentaje cambio la forma de impartir clases por parte de los docentes, y el porcentaje cambio la forma de clases por parte de los docentes.

El sistema educativo en el Ecuador debe invertir en el desarrollo profesional de los docentes, especialmente en tecnologías de la información y la comunicación (TIC) y una pedagogía eficaz, considerando el escenario actual. Hacer que la enseñanza en línea sea creativa, innovadora e interactiva a través de herramientas fáciles de usar es la otra área de investigación y desarrollo, esto ayudaría y prepararía al sistema educativo para tales incertidumbres en el futuro. 


\section{Referencias}

Acosta, A. (2020). El coronavirus en los tiempos del Ecuador. Análisis Carolina, 1-19. https://doi.org/10.33960/ ac 23.2020

Acuña, M. (2021). América latina. Entre la nueva realidad y las viejas desigualdades. Telos Revista de Estudios Interdisciplinarios En Ciencias Sociales, 23(1), 129-140. https://doi.org/10.36390/telos231.10

Andarwulan, T., Al Fajri, T., \& Damayanti, G. (2021). Elementary teachers' readiness toward the online learning policy in the new normal era during Covid-19. International Journal of Instruction, 14(3), 771-786. https:// doi.org/10.29333/iji.2021.14345a

Guillermina, B. P. (2014). Metodología de la Investigación (C. Javier Enrique (ed.); Primera). Grupo Editorial Patria.

Hernández-Sampieri, R., \& Mendoza, C. (2018). Metodología de la investigación (M. Rocha (ed.); Primera). Mc Graw Hill Education.

Hernández Sampieri, R., Fernández Collado, C., \& Baptista Lucio, P. (2014). Metodología de la investigación (McGrawHill (ed.); Sexta Edic). McGrawHill.

Pacheco, J. (2020). The "new normal" in education. Prospects. https://doi.org/10.1007/s11125-020-09521-x

Plá, S., Buenfil, R., Zabalgoitia, M., Gallardo, A., Constante, A., De la Cruz, G., González, E., \& Orozco, B. (2020). La educación entre la COVID-19 y el emerger de la nueva normalidad. Perfiles Educativos, 42(170), 1-72. https://doi.org/10.22201/iisue.24486167e.2020.170.60181

Prince, Á. (2021). Aulas híbridas: Escenarios para transformación educativa dentro de la nueva normalidad. Podium, (39), 103-120. https://doi.org/10.31095/podium.2021.39.7

Tejedor, S., Cervi, L., Tusa, F., \& Parola, A. (2020). Educación en tiempos de pandemia: reflexiones de alumnos y profesores sobre la enseñanza virtual universitaria en España, Italia y Ecuador. Revista Latina, (78), 1-21. https://doi.org/10.4185/rlcs-2020-1466

Tria, J. (2020). The COVID-19 Pandemic through the Lens of Education in the Philippines: The New Normal. International Journal of Professional Development, Learners and Learning, 1(1), 2-4. https://doi.org/10.30935/ ijpdll//8311

Vargas Jiménez, K. (2020). Enseñanza aprendizaje virtual en tiempos de pandemia [Tesis de grado, Universidad Estatal del Sur de Manabí]. 
Copyright (c) 2021 Franklin Gregorio Macias Arroyo, Lorgia Patricia Astudillo Erazo y Silvino Alejandro Macías Arroyo

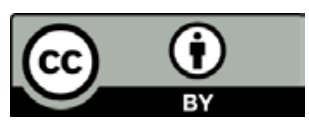

Este texto está protegido bajo una licencia internacional Creative Commons 4.0.

Usted es libre para Compartir-copiar y redistribuir el material en cualquier medio o formato - y Adaptar el documento - remezclar, transformar y crear a partir del material-para cualquier propósito, incluso para fines comerciales, siempre que cumpla las condiciones de Atribución. Usted debe dar crédito a la obra original de manera adecuada, proporcionar un enlace a la licencia, e indicar si se han realizado cambios. Puede hacerlo en cualquier forma razonable, pero no de forma tal que sugiera que tiene el apoyo del licenciante o lo recibe por el uso que hace de la obra.

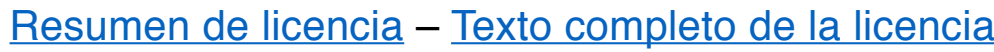

